\title{
A MECHANICAL MODEL FOR THE BUCKLING OF UNILATERALLY CONSTRAINED RECTANGULAR PLATES
}

\author{
Khaled W. Shahwan and Anthony M. WaAs \\ Composite Structures Laboratory, Department of Aerospace Engineering, The University of \\ Michigan, Ann Arbor, MI 48109-2140, U.S.A.
}

(Received 2 January 1993 ; in revised form 4 June 1993)

\begin{abstract}
A mechanical model is described for the problem of buckling of unilaterally constrained, finite, rectangular plates. Due to the nature of the imposed constraint on the plate's lateral deflection, $w$, solving for the buckling load required the solution of a nonlinear partial differential equation in $w$. While the plates were modeled along the lines of classical plate theory, the nonlinearity arose from the fact that the plates were attached to nonlinear elastic foundations exhibiting a deformation sign dependent force-displacement relationship. This feature was introduced to model the unilateral constraint. The influence of different boundary conditions, material orthotropy and transverse load distributions was investigated. For each case, the weak form of the governing differential equation was solved via the Galerkin's method. Investigations of the buckling loads of rectangular plates attached to such foundations and subjected to a uniform inplane stress field showed the validity of this approach for the cases investigated and compared to some previous exact results reported in the literature.
\end{abstract}

\section{NOMENCLATURE}

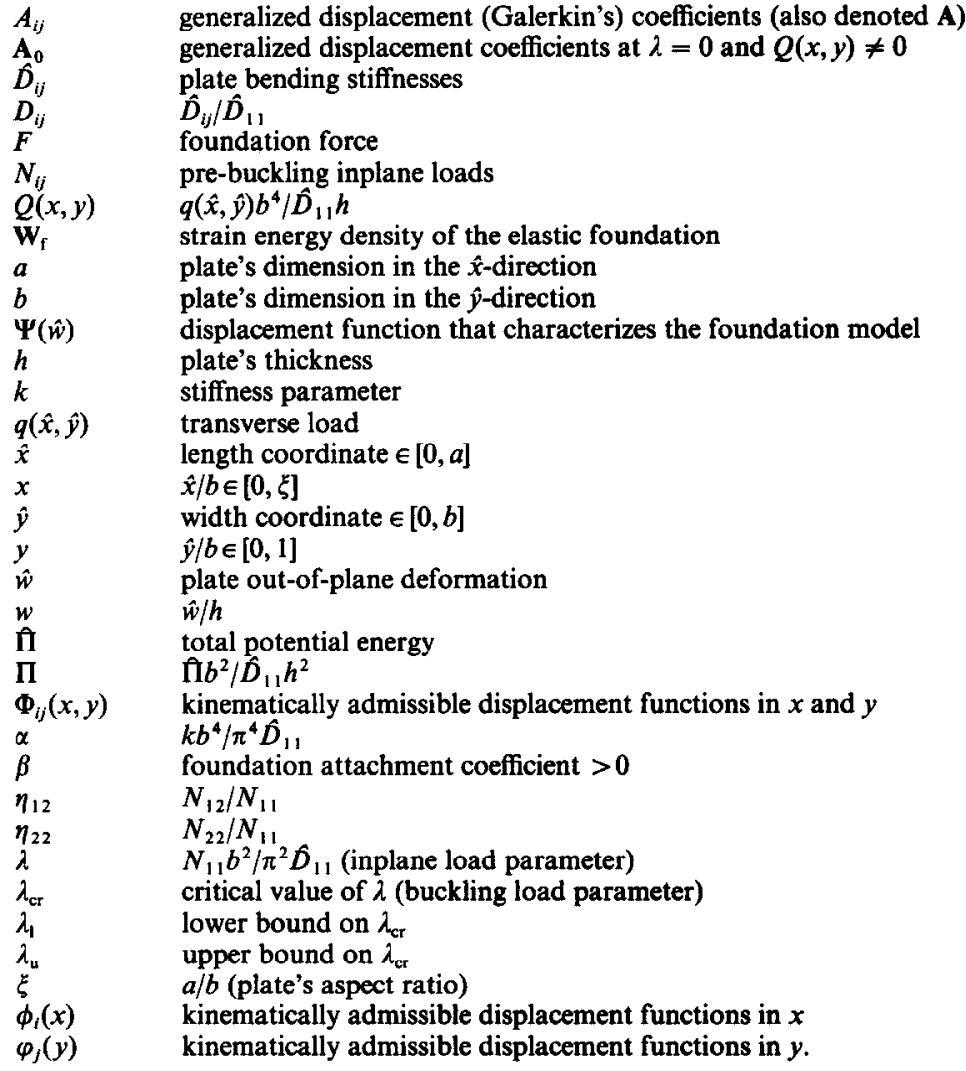

\section{INTRODUCTION}

In this paper we consider a mechanical model and an appropriate solution method for the critical loading conditions of a finite, rectangular and linear elastic plate that is unilaterally 


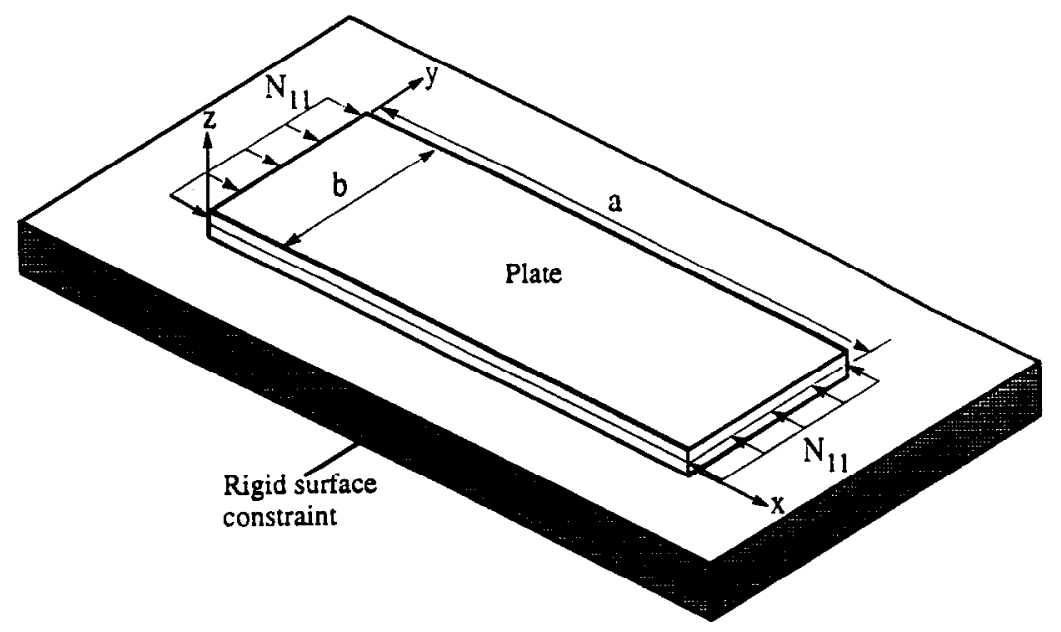

Fig. 1. Thin elastic plate constrained by a rigid surface and under the action of an applied inplane uniform load.

constrained by the presence of a rigid surface parallel to the plate's undeformed middle plane as shown in Fig. 1. The distance between the rigid surface and the plate's middle plane is taken to be half the plate's thickness, implying that a gap does not exist. The plate is subjected to transverse distributed loading as well as a uniform inplane stress field. The plate is modeled along the lines of classical plate theory employing the Kirchhoff-Love hypothesis.

The model description in the preceding paragraph is characteristic of a variety of practical situations. For example, consider the problem of near-surface delamination (disbond) buckling in laminated composite plates. Due to the out-of-plane thickness ratio of the delamination (thin plate) to that of the sublaminate (parent substrate), the sublaminate essentially acts like a rigid surface constraining the plate's out-of-plane deformations to be of one sign. This constraint is usually avoided indirectly by either modeling the thin plate as a wide column or as an axisymmetric circular plate or annulus (Chai et al., 1981 ; Bottega and Maewal, 1983; Sallam and Simitses, 1985; Bruno and Grimaldi, 1990; Barbero and Reddy, 1991), which are essentially one-dimensional models. However, it is to be noted that except for certain inner/outer radii ratios, annuli can exhibit a nonaxisymmetric behavior (Majumdar, 1971 ; Fu and Waas, 1992). By resorting to one-dimensional models, the governing partial differential equations are reduced to ordinary differential equations. This implies that the eigenmode corresponding to the lowest eigenvalue is of one sign, hence, no contact occurs (within the boundaries) between the delamination and the sublaminate. An analysis of the one-dimensional case (Chai et al., 1981) where the sublaminate has some finite bending rigidity, reveals that certain buckling configurations exist in which contact conditions occur. The axisymmetric upheaval buckling of a heavy plate in unilateral contact with a rigid subgrade was presented in Hobbs (1990).

One common simplification when addressing these types of problems is the assumption that the medium, being a beam or a plate, is infinite. The buckling of a unilaterally constrained infinite beam (thin strip) was addressed by many investigators (Allan, 1968; Anderson, 1972; Yun and Kyriakides, 1983 ; Wang, 1984a,b ; Hobbs, 1985 ; Roorda, 1988 ; Plaut and Mróz, 1992), and it was shown that such a system is completely imperfectionsensitive, with theoretically infinite buckling load in the absence of imperfections or weightlessness. An analysis to extend the classic variational theory for eigenvalue problems so as to define the Euler critical load of a unilaterally constrained beam is presented in Villaggio (1979). The variational formulation also provided a method of bounding the buckling load by comparison, and a few important theorems and remarks were stated. Using an elastica approach, Soong and Choi (1986) derived equations for continuous contact between a beam and its boundary, as well as multiple discrete point contacts. They presented examples 
in which the elastica curvature assumes values which are less than the curvature of the restraining boundary, thus resulting in line contact. A finite element solution was used by Stein and Wriggers (1984) with an updated Lagrangean formulation to investigate the stability of rods with unilateral constraints. These authors showed good agreement in comparing their solutions to those available in the literature.

Similar issues relating to the behavior of unilaterally constrained plates have been considered by other researchers in different contexts. Motivated by a problem that arises in magnetic tape recording, Benson (1991) used classical plate theory to study plate tenting with one-sided constraint using an energy minimization formulation. A more indepth study on unilateral boundary value problems as related to the nonlinear theory of plates was reported in Naumann $(1975,1977)$. This author presents a variational formulation along with an existence theorem and a uniqueness result for a variational solution for thin plates under normal load. The existence of equilibrium states of such plates is also studied.

The buckling behavior of an infinitely long, simply-supported isotropic plate is presented in Seide (1958). Attaching the plate to a tensionless foundation and subjecting it to a far field uniaxial compressive load, he presented results for the buckling load as well as the buckling wave length. Using a limiting process, the solution for a unilaterally constrained simply-supported infinite plate was recovered from the exact solutions of the governing differential equations. Such a solution showed a 33\% increase in the buckling load when the plate was unilaterally constrained. Using a similar formulation, Shahwan and Waas (1991), obtained similar results for specially orthotropic plates as well as approximate results for plates that have clamped-free boundary conditions on their unloaded edges. They reported values for the \% increase in the buckling load for four types of specially orthotropic plates.

One of the earliest works that addressed the issue of unilateral constraints as related to the problem of two-dimensional delamination buckling was reported by Chai and Babcock (1985). Modeling the delamination as a thin elliptic plate, and based on a rather limited number of assumed admissible Rayleigh-Ritz displacement terms, they studied the dependence of overlap conditions on ellipse aspect ratio and load level. More recently, the buckling and post-buckling of elliptical delaminations was investigated by Chai $(1990 \mathrm{a}, \mathrm{b})$, who carried out a simplified contact analysis by limiting the contact regions to isolated points. Although such an assumption resulted in an accuracy within $\pm 30 \%$, it would not hold in cases where the geometry, boundary conditions and loading are of a more complicated nature so that surface rather than point contact would dominate the response.

The buckling of thin plates using von Kármán plate theory in a variational inequality formulation was presented in Do $(1976,1977)$ and Kubrusly and Oden (1981). Using a variational principle with penalty in a finite element formulation, Ohtake et al. $(1980 \mathrm{a}, \mathrm{b})$ provided a more convenient basis for computational methods by introducing a penalty term and adding it to the potential energy. In effect, this term is identically zero whenever the plate's deformation $w$ is not in contact with the rigid constraint, and greater than zero otherwise.

Issues pertaining to the equilibrium and stability of discrete one-way structural systems were dealt with in a general manner by Burgess (1971a). This author also presented an analysis that showed that the discrete method converges to the continuum solution by studying a radially constrained imperfect ring (Burgess, 1971b). In these works the notion of a critical state is classified and the evaluation of the buckling load was carried out in the context of a postbuckling analysis.

In the present work, the problem of unilateral constraint is modeled by introducing a nonlinear elastic foundation that influences the plate's out-of-plane deformation as shown in Fig. 2. This is a feature of the modeling that is introduced and not necessarily of the problem at hand. Indeed, from this point of view, the present work is similar in spirit to the approach adopted in Seide (1958).

\section{PROBLEM FORMULATION}

In order to account for the physical constraint imposed on the plate's buckling displacements, a nonlinear elastic foundation model that exhibits a deformation sign dependent 


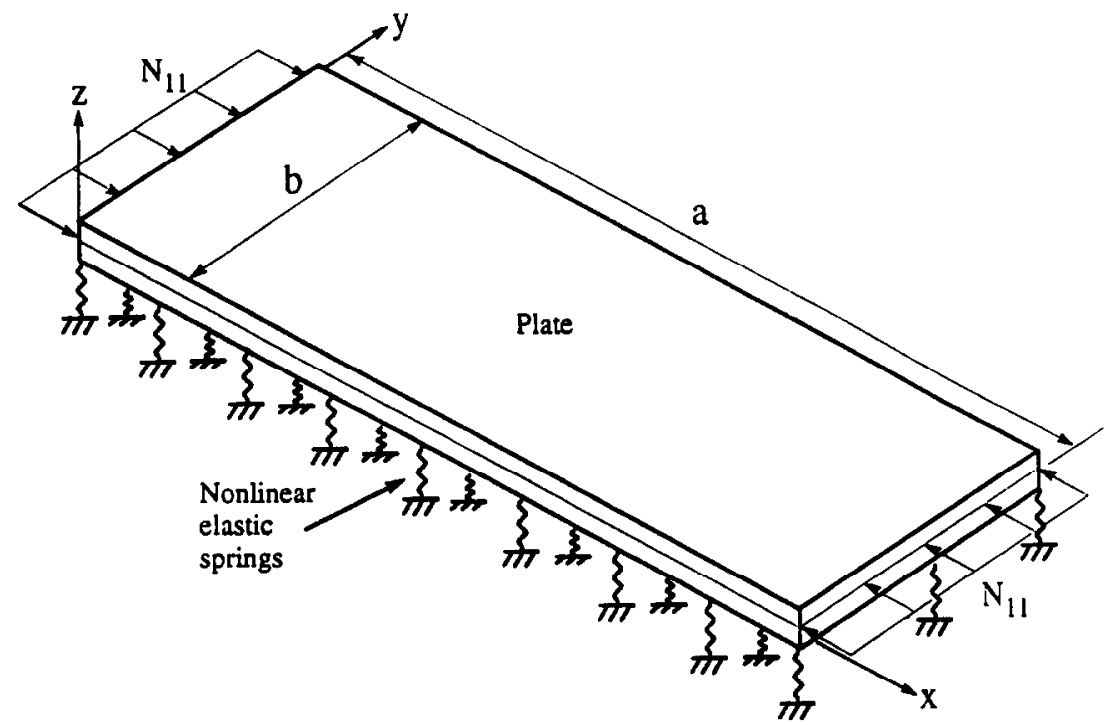

Fig. 2. Modeling the rigid surface constraint as a nonlinear elastic foundation (springs).

force-displacement relationship was implemented. Such types of nonlinearities present analytical difficulties in that an exact closed-form solution cannot be easily obtained if at all. As such, an approximate method of solution must be followed to formulate the equations governing the plate's response. From the expression of the total potential energy (1), one can operate either on its functional form directly (e.g. the Rayleigh-Ritz method), or on its first variation form (e.g. the Galerkin method) :

$$
\begin{aligned}
\hat{\Pi}=\frac{1}{2} \int_{0}^{b} \int_{0}^{a}\left[\hat{D}_{11} \hat{w}_{, \hat{x} \hat{x}}^{2}+2 \hat{D}_{12} \hat{w}_{, \hat{x} x} \hat{w}_{, \hat{y} \hat{y}}+\hat{D}_{22} \hat{w}_{\hat{y} \hat{y}}^{2}+4\left(\hat{D}_{16} \hat{w}_{, \hat{x} \hat{x}}+\hat{D}_{26} \hat{w}_{, \hat{y} \hat{y}}\right) \hat{w}_{, \hat{x} \hat{y}}\right. \\
\left.+4 \hat{D}_{66} \hat{w}_{, \hat{x} \hat{y}}^{2}\right] \mathrm{d} \hat{x} \mathrm{~d} \hat{y}-\frac{1}{2} \int_{0}^{b} \int_{0}^{a}\left[N_{11} \hat{w}_{, \hat{x}}^{2}+2 N_{12} \hat{w}_{, \hat{x}} \hat{w}_{, \hat{y}}+N_{22} \hat{w}_{, \hat{y}}^{2}\right] \mathrm{d} \hat{x} \mathrm{~d} \hat{y} \\
\quad-\int_{0}^{b} \int_{0}^{a} q(\hat{x}, \hat{y}) \hat{w} \mathrm{~d} \hat{x} \mathrm{~d} \hat{y}+\int_{0}^{b} \int_{0}^{a} \mathbf{W}_{\mathrm{f}} \mathrm{d} \hat{x} \mathrm{~d} \hat{y}
\end{aligned}
$$

The elastic foundation's strain energy density functional, $\mathbf{W}_{\mathrm{f}}$, is defined as,

$$
\mathbf{W}_{\mathrm{f}}=\int k \Psi(\hat{w}) \mathrm{d} \hat{w}
$$

In the case of a linear elastic foundation, $\Psi(\hat{w})=\hat{w}$, and hence $\mathbf{W}_{\mathrm{f}}=\frac{1}{2} k \hat{w}^{2}$, where $k$ is the linear foundation stiffness. Nondimensionalizing (1) will result in the following expression for the total potential energy :

$$
\begin{aligned}
\Pi=\frac{1}{2} \int_{0}^{1} \int_{0}^{\xi}\left[w_{, x x}^{2}+2 D_{12} w_{, x x} w_{, y y}+D_{22} w_{, y y}^{2}+4\left(D_{16} w_{, x x}+D_{26} w_{, y y}\right) w_{, x y}\right. \\
\left.+4 D_{66} w_{, x y}^{2}\right] \mathrm{d} x \mathrm{~d} y-\frac{1}{2} \int_{0}^{1} \int_{0}^{\xi} \lambda \pi^{2}\left[w_{, x}^{2}+2 \eta_{12} w_{, x} w_{, y}+\eta_{22} w_{, y}^{2}\right] \mathrm{d} x \mathrm{~d} y \\
-\int_{0}^{1} \int_{0}^{\xi} Q(x, y) w \mathrm{~d} x \mathrm{~d} y+\int_{0}^{1} \int_{0}^{\xi} \alpha \pi^{4}\left(\int \Psi(w) \mathrm{d} w\right) \mathrm{d} x \mathrm{~d} y
\end{aligned}
$$


Calculating the first variation of $\Pi$ and applying the divergence theorem yields the following variational equation:

$$
\begin{aligned}
\delta \Pi= & \int_{0}^{1} \int_{0}^{\xi}\left[w_{, x x x x}+2\left(D_{12}+2 D_{66}\right) w_{, x x y y}+D_{22} w_{, y y y y}+4\left(D_{16} w_{, x x x y}+D_{26} w_{, x y y y}\right)\right. \\
& \left.+\lambda \pi^{2}\left(w_{, x x}+2 \eta_{12} w_{, x y}+\eta_{22} w_{, y y}\right)+\alpha \pi^{4} \Psi(w)-Q(x, y)\right] \delta w \mathrm{~d} x \mathrm{~d} y \\
& -\int_{0}^{1}\left(M_{x x} \delta w_{, x}+M_{x y} \delta w_{, y}-V_{x} \delta w\right)_{x=0}^{x=\xi} \mathrm{d} y-\int_{0}^{\xi}\left(M_{x y} \delta w_{, x}+M_{y y} \delta w_{, y}-V_{y} \delta w\right)_{y=0}^{y=1} \mathrm{~d} x,
\end{aligned}
$$

where $M_{x x}, M_{y y}, M_{x y}, V_{x}$ and $V_{y}$ are the nondimensional moments and shear forces at the plate's boundary and in terms of $w(x, y)$ are given in the following equations:

$$
\begin{aligned}
M_{x x}= & -\left(w_{, x x}+D_{12} w_{, y y}+2 D_{16} w_{, x y}\right), \\
M_{y y}= & -\left(D_{12} w_{, x x}+D_{22} w_{, y y}+2 D_{26} w_{, x y}\right), \\
M_{x y}= & -\left(D_{16} w_{, x x}+D_{26} w_{, y y}+2 D_{66} w_{, x y}\right), \\
V_{x}= & -\left[w_{, x x x}+3 D_{16} w_{, x x y}+\left(D_{12}+2 D_{66}\right) w_{, x y y}\right. \\
& \left.+D_{26} w_{, y y y}+\lambda \pi^{2}\left(w_{, x}+\eta_{12} w_{, y}\right)\right], \\
V_{y}= & -\left[D_{16} w_{, x x x}+\left(D_{12}+2 D_{66}\right) w_{, x x y}\right. \\
& \left.+3 D_{26} w_{, x y y}+D_{22} w_{, y y y}+\lambda \pi^{2}\left(\eta_{12} w_{, x}+\eta_{22} w_{, y}\right)\right] .
\end{aligned}
$$

Investigations of equilibrium states requires the necessary and sufficient condition that the total potential energy $\Pi$ be stationary, hence, the vanishing of its first variation,

$$
\delta \Pi=0 .
$$

From (4), (5) and (6) the governing nonlinear differential cquation can be extracted along with the boundary conditions. A closed-form solution of the differential equation is highly dependent on the form of $\Psi(w)$. While for nonlinear $\Psi(w)$ such a solution is near impossible, it is easily obtainable if $\Psi(w)$ is linear in $w$ and if the boundary conditions are of a certain type and combination. Hence, for nonlinear $\Psi(w)$, one has to resort to approximate methods such as the finite element method, the Rayleigh-Ritz method, or Galerkin's method, to mention a few. In this study it was decided to employ Galerkin's method, and in order to carry out the solution procedure, kinematically admissible global displacement functions must be assumed. It is important to note that although the plate is unilaterally constrained, such a constraint does not play any role in choosing these functions and as mentioned earlier, this constraint condition will be accounted for indirectly via the nonlinearity of the elastic foundation model. Further, the out-of-plane displacement field $w$ can be assumed to be of a separable form where shape functions in $x$ are multiplied by those in $y$. These functions can be chosen to be the buckling and/or free vibrational eigenmodes of beams and/or plates having the same kinematic boundary conditions. In the forthcoming analysis, the out-of-plane displacement functions $w(x, y)$ were chosen to have the following form :

$$
w(x, y)=\sum_{i=1}^{M} \sum_{j=1}^{N} A_{i j} \Phi_{i j}(x, y)
$$

where $\Phi_{i j}(x, y)$ has the following separable form : 


$$
\Phi_{i j}(x, y)=\phi_{i}(x) \varphi_{j}(y)
$$

$\phi_{i}(x)$ and $\varphi_{j}(y)$ must satisfy their corresponding plate's kinematic boundary conditions. The first variation of $w(x, y)$ with respect to the generalized displacement coefficients $A_{i j}$ is,

$$
\delta w(x, y)=\sum_{i=1}^{M} \sum_{j=1}^{N} \delta A_{i j} \Phi_{i j}(x, y)
$$

Substituting (9) in (4) and in conjunction with the statement of stationary total potential energy (6) one arrives at the following set of $M \times N$ algebraic equations in the $M \times N$ unknowns $A_{i j}$ :

$$
\begin{aligned}
\int_{0}^{1} \int_{0}^{\xi} & {\left[w_{, x x x x x}+2\left(D_{12}+2 D_{66}\right) w_{, x x y y}+D_{22} w_{, y y j y y}\right.} \\
& +4\left(D_{16} w_{, x x x y}+D_{26} w_{, x y y y}\right)+\lambda \pi^{2}\left(w_{, x x}+2 \eta_{12} w_{, x y}\right. \\
& \left.\left.+\eta_{22} w_{, y y}\right)+\alpha \pi^{4} \Psi(w)-Q(x, y)\right] \Phi_{i j}(x, y) \mathrm{d} x \mathrm{~d} y \\
& -\int_{0}^{1}\left(M_{x x} \Phi_{i j, x}+M_{x y} \Phi_{i j, y}-V_{x} \Phi_{i j}\right)_{x=0}^{x=\xi} \mathrm{d} y \\
& -\int_{0}^{\xi}\left(M_{x y} \Phi_{i j, x}+M_{y y} \Phi_{i j, y}-V_{y} \Phi_{i j}\right)_{y=0}^{y=1} \mathrm{~d} x=0 .
\end{aligned}
$$

For a prescribed $\Psi(w)$, one can determine the buckling load parameter $\lambda_{\mathrm{cr}}$ as well as the generalized displacements $A_{i j}$ using an incremental load approach. The solution algorithm increments the inplane load parameter $\lambda$ and monitors the determinant of the incremental stiffness matrix. $\lambda_{\mathrm{cr}}$ was obtained when the incremental stiffness matrix became singular (or near singular). Such an approach is used frequently in the analysis of nonlinear problems.

The type of foundation model needed to incorporate the physics of unilaterally constrained plates should exhibit a force-displacement relationship that is deformation sign dependent, hence, the foundation was modeled as extensional springs having such a relationship. Such models have been considered by many investigators in the treatment of beams and plates resting on nonlinear foundations (Tsai and Westmann, 1967; Farshad and Shahinpoor, 1972; Celep, 1988). In these studies, the sgn, Dirac delta as well as Heaviside step functions were used to describe the bimodulus nature of the elastic foundation (bimodulus in the sense that compression stiffness is different than that for tension). In this study, a model that utilizes the switching property of the tanh function was used. The forcedisplacement relationship for this model is given below as,

$$
F=\alpha \Psi(w),
$$

where

$$
\Psi(w)=w\left[\frac{1}{2}(1-\tanh (\beta w))\right]
$$

$\alpha$ is a nondimensional stiffness parameter, $w$ is the normalized deformation, $\beta$ is a spring (foundation) attachment coefficient that is $>0$. The foundation attachment can be controlled by changing the value of the parameter $\beta$. Large values of $\beta$ imply less attachment 


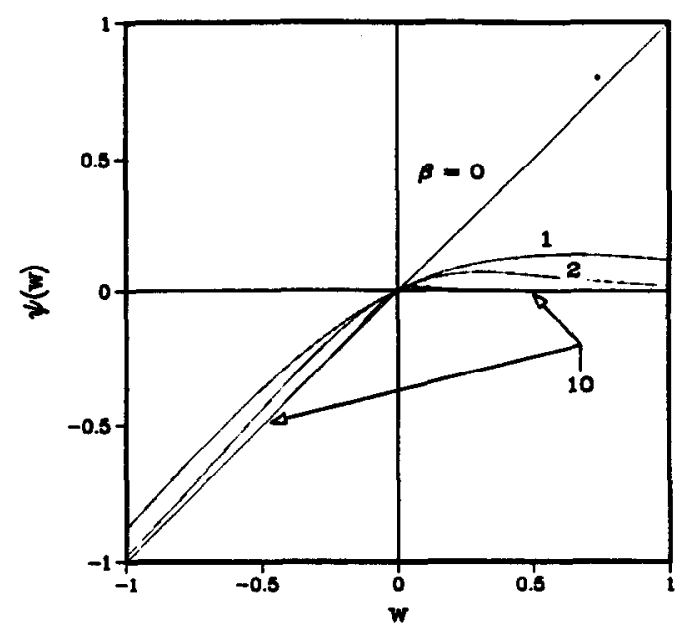

Fig. 3. Displacement function that characterizes the foundation model $\Psi(w)$ as a function of $w$ for different values of $\beta$.

as shown in Fig. 3. Theoretically, $\beta=\infty$ implies that the foundation is fully unattached (tensionless), while $\beta=0$ implies the foundation is fully attached (note that if $\beta=0$ the $\frac{1}{2}$ factor in (12) should be replaced by 1 in order to recover the linear case where $\Psi=w$ ). Furthermore, while foundation stiffness can be increased to larger values by increasing $\alpha$, increasing $\beta$ will result in a decrease in the ratio of the tension/compression stiffnesses.

It is worthwhile noting that although the nonlinearity in the physical problem is geometric, arising from the constraints imposed on the plate's behavior, the nonlinearity in the governing equations arises from the elastic foundation's constitutive model. Notice that this is a feature of the mechanical model that is employed in the present work.

The type of nonlinearity in the physical problem invalidates any linearization procedure of the governing equations about the trivial state. As such, the fully nonlinear equations must be solved which introduces complications that are associated with the foundation's force-displacement relationships (11) and (12) where linearization of $\tanh (\beta w)$ for large $\beta$ does not reflect the intended physical situation. The inability to linearize the governing equations without substantially changing the physics of the problem is a distinguishing feature of this class of problems.

\section{RESULTS AND DISCUSSION}

While generic buckling curves for uniaxially loaded rectangular plates are available in the literature (Gerard and Becker, 1957; Brunelle and Oyibo, 1983), such results are nonexistent for unilaterally constrained plates. Hence, in this study the effort was concentrated on trying to generate such curves for plates having different boundary conditions and subjected to a uniaxial stress field. For uniaxial loading, $\eta_{12}=\eta_{22}=0$. In problems of the type presented here, it is often easier to obtain the buckling loads by treating a response problem and determining the value of the inplane load parameter $(\lambda)$ corresponding to a rapid growth in the response. More specifically, the notion of a rapid growth in the context of the numerical solution that we have obtained is clearly discussed later on. Thus, values of buckling load presented here were obtained, and the term buckling load is used, in this context.

In carrying out the solution process, the value of $\beta$ in (12) posed a computational drawback. Although large values for $\alpha$ as well as $\beta$ are required in simulating the presence of the rigid surface, they cannot be chosen arbitrarily large. It was observed that while $\alpha=100$ ("high stiffness" foundation) yields adequate and physically admissible results independent of $w$, the value of $\beta$ was dependent on $w$ so as to maintain the product $\beta w$ as large as possible for all values of $w$. Further, assigning a large constant value of $\beta$, led, in some cases, to numerical difficulties that were encountered in the incremental method 


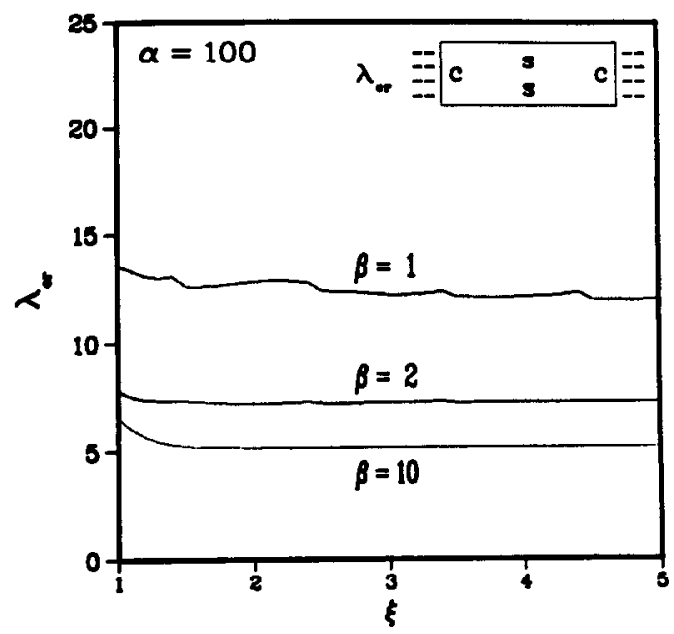

Fig. 4. The influence of the foundation (springs) attachment coefficient $\beta$ on the value of the buckling load parameter $\lambda_{\text {cr }}$ for an isotropic plate with "ccss" boundary conditions.

algorithm. In these cases, a physically inadmissible result such as plate penetration into the foundation was found to exist. An improvement on this foundation model (12) that eliminates such dependency can be achieved by casting (12) in the following form:

$$
\Psi(w)=w\left[\frac{1}{2}\left(1-\tanh \left(\beta \frac{w}{\|w\|}\right)\right)\right]
$$

Using the above form of $\Psi(w)$, and after investigating a wide range of problems, it was found that $\beta=10$ is an adequate value for an attachment coefficient representing the unattached situation. Figure 4 demonstrates the dependence of $\lambda_{\mathrm{cr}}$ on $\beta$ as a function of $\xi$ for plates having clamped and simply-supported boundary conditions along their loaded and unloaded edges, respectively. Curves for $\beta>10$ are indistinguishable from the $\beta=10$ curve.

A simplification to the solution algorithm rests in the fact that, for a given case where $\alpha$ and $\beta$ are $>0, \lambda_{\mathrm{cr}}$ is bounded from above and below. The lower bound $\lambda_{1}$ corresponds to $\lambda_{\mathrm{cr}}$ when $\alpha=0$, while the upper bound $\lambda_{\mathrm{u}}$ corresponds to $\lambda_{\mathrm{cr}}$ when $\beta=0$. Such bounds were used as guides in the incremental method and it was noticed that, in general, for unilaterally constrained plates, $\lambda_{\mathrm{cr}}$ is closer to $\lambda_{1}$ than $\lambda_{\mathrm{u}}$ indicating that the rigid surface constraint does not increase the buckling load significantly (Seide, 1958; Shahwan and Waas, 1991). For an isotropic, simply-supported (along the unloaded edges) infinite plate, such an increase was found to be $33 \%$.

By using Galerkin's method the governing nonlinear differential equation was reduced to a set of $M \times N$ nonlinear algebraic equations (10) whose solution was carried out iteratively using the Levenberg-Marquardt algorithm (denoted as LMP) as modified by Powell (1970). For a system of nonlinear algebraic equations $\mathbf{f}(\mathbf{A})=0$ the algorithm is given by

$$
\mathbf{A}_{k+1}=\mathbf{A}_{k}-\left[\rho_{k} \mathbf{I}+\mathbf{J}_{k}^{\mathrm{T}} \mathbf{J}_{k}\right]^{-1} \mathbf{J}_{k}^{\mathrm{T}} \mathbf{f}\left(\mathbf{A}_{k}\right),
$$

where $\mathbf{J}_{k}$ is the Jacobian at the $k$ th iteration given by

$$
\mathbf{J}_{k}=\frac{\partial \mathbf{f}}{\partial \mathbf{A}}\left(\mathbf{A}_{k}\right)
$$

and $\mathbf{A}_{k}$ is the current, known, approximate value of the vector of unknowns $A$ and $\rho_{k}$ is a scalar that is selected based on a methodology given in Powell (1970). Although this 


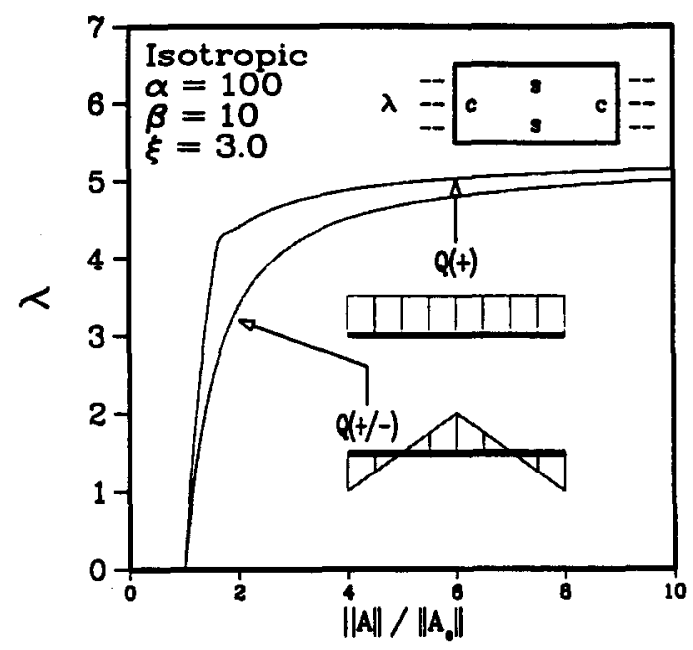

Fig. 5. Response curves for two different transverse loading $Q(x, y)$ distributions, for an isotropic "ccss" plate of aspect ratio $\xi=3$.

algorithm was the main algorithm used in obtaining solutions for $\mathbf{A}$, for some cases and for $\lambda$ near $\lambda_{\text {cr }}$, the algorithm didn't converge and Newton's method was temporarily employed. Such a situation did not occur frequently. In those few instances, we traced the reason for this lack of convergence to be associated with the evaluation of the Jacobian. In Newton's method, the Jacobian at the $k$ th iteration $\left(\mathbf{J}_{k}\right)$ is evaluated with a pre-assigned step size, while, in the LMP method, the $\mathbf{J}_{k}$ is updated automatically with step size that depends on $\mathbf{A}_{k}$ as well as the change in the Jacobian from the previous two iterations.

\section{Dependency of $\lambda_{c r}$ on the transverse load $\mathrm{Q}(\mathrm{x}, \mathrm{y})$}

In the absence of $Q(x, y)$ and since the trivial solution $(w=0)$ is an admissible solution for all values of $\lambda$, the solution of the governing equations constitutes the determination of the eigenvalues $\lambda_{\mathrm{cr}}$ and their corresponding eigenmodes. But due to the nonlinearity of these equations, such a system falls into the category of a nonlinear eigenvalue problem. As a simplification to the solution process a transverse load $Q(x, y)$ was added which converts the problem from being a nonlinear eigenvalue problem to a nonlinear response problem. In order to establish that the results for $\lambda_{\mathrm{cr}}$ are not significantly influenced by the magnitude and distribution of $Q(x, y)$, different cases were investigated where the sign and magnitude as well as the functional distribution throughout the plate were varied. Although the "response curves" (curves representing $\lambda$ vs normalized magnitude of the generalized displacements vector $\left.\|\mathbf{A}\| /\left\|\mathbf{A}_{0}\right\|\right)$ were not significantly influenced by the magnitude of $Q(x, y)$, they were dependent on its sign distribution. However, the value of $\lambda$ at which the plate response appeared to increase without bound (approaching buckling) was independent of $Q(x, y)$ as expected. If $Q(x, y)=c$, where $c$ is a constant that is $<0$ (i.e. $Q$ is pushing the plate against the foundation), the value of $\lambda_{\mathrm{cr}}$ was equal to $\infty$. If, on the other hand, $c>0$ (i.e. $Q$ is pulling the plate away from the foundation), the value of $\lambda_{\mathrm{cr}}$ was finite yielding valid results. However, the latter situation has an interesting feature that can be seen in Fig. 5. In this figure, the response curves for a plate that is clamped along the loaded edges and simply supported otherwise is under the action of two types of transverse loads. One curve represents the case where $c>0$ while the other represents the case where $Q(x, y)$ is a symmetric distribution (symmetry with respect to the center line $x=\xi / 2$ ). In the former, a decrease and then a sudden increase in the slope at some value of $\lambda<\lambda_{\text {cr }}$ is observed. This can be attributed to the plate "touching" the foundation and immediately experiencing additional stiffness due to the foundation. On the other hand, in the latter case where the transverse load has negative as well as positive distributions, "touching" is already present, 


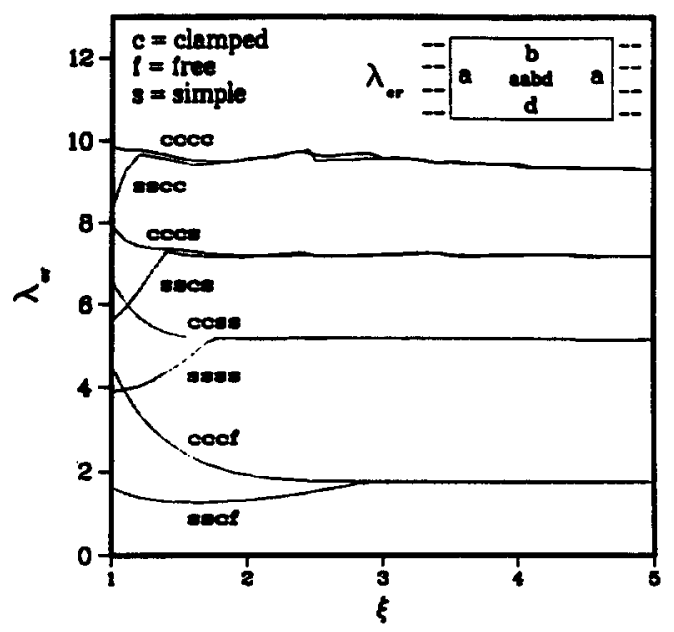

Fig. 6. Buckling load parameter $\lambda_{\mathrm{cr}}$ as a function of the plate's aspect ratio $\xi$ for isotropic plates with different boundary conditions on their loaded and unloaded edges.

prior to the application of the axial load. Hence, this sudden slope increase is not strongly present. Further, although such difference between these two curves is strongly present for intermediate values of $\lambda$, it vanishes at the early stages of loading $(\lambda)$ increments as well as when $\lambda$ approaches the eigenvalue $\left(\lambda_{\text {cr }}\right)$. From this figure, it is also seen that as the magnitude of the normalized generalized displacement vector $\|\mathbf{A}\| /\left\|\mathbf{A}_{0}\right\|$ tends to large values (exceeding 10), the slope of the response curves tends to zero. In order to determine $\lambda_{\text {cr }}$, we plotted several response curves for each case, and in every case we found all the response curves to be within $1 \%$ of each other when $\|\mathbf{A}\| /\left\|\mathbf{A}_{0}\right\|$ was, at most, approximately 20 . The load corresponding to this limit was chosen as $\lambda_{\mathrm{cr}}$. Clearly, for some plates this limit was reached much earlier $\left(\|\mathbf{A}\| /\left\|\mathbf{A}_{0}\right\|\right.$ approximately 10). It is worthwhile pointing out that in the presence of $Q(x, y)$, the obtained $\lambda_{\text {cr }}$ will always be an underestimate of the exact $\lambda_{\text {cr }}$ value (eigenvalue) that would have been obtained by setting $Q(x, y)=0$, provided a sufficient number of Galerkin terms are used. Although this difference should approach 0 as $Q(x, y)$ approaches 0 , its value was kept to a minimum (i.e. $Q(x, y)$ was never set to 0 ).

\section{Dependency of $\lambda_{c r}$ on the plate's aspect ratio $\xi$}

Figures 6 and 7 show the dependency of $\lambda_{\text {cr }}$ on the plate's aspect ratio $\xi$ for different types of boundary conditions on the loaded and unloaded edges as well as for different

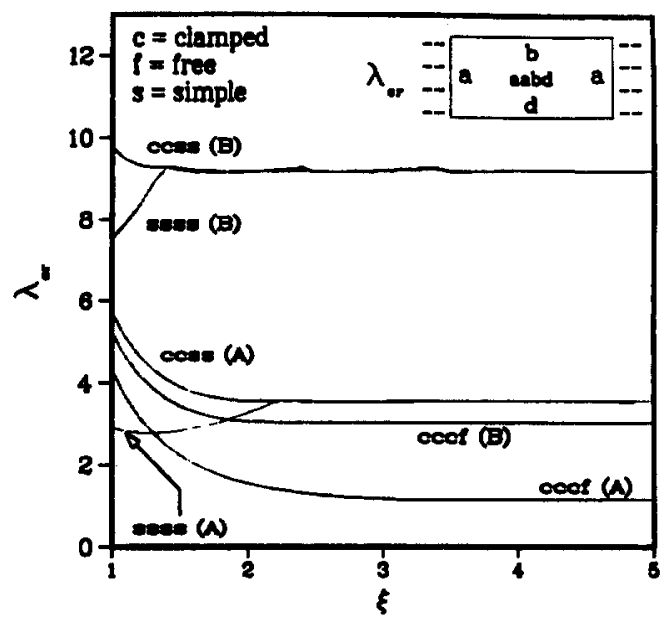

Fig. 7. Buckling load parameter $\lambda_{\text {cr }}$ as a function of the plate's aspect ratio $\xi$ for two types of orthotropic plates (A and B) with different boundary conditions on their loaded and unloaded edges. 
Table 1. Limiting values of the buckling load parameter $\left(\lambda_{\mathrm{cr}}\right)$ as $\xi \rightarrow$ $\infty$ for uniaxially loaded isotropic plates

\begin{tabular}{|c|c|c|c|c|}
\hline & \multicolumn{4}{|c|}{ Boundary conditions $\dagger$} \\
\hline Loaded edges $\ddagger$ & \multicolumn{4}{|c|}{ cc or ss } \\
\hline Unloaded edges§ & $\mathrm{cc}$ & cs & ss & cf \\
\hline$\lambda_{\text {cr }}$ & 9.30 & 7.22 & $5.25 \|$ & 1.75 \\
\hline
\end{tabular}

types of materials. As expected, the dependence of $\lambda_{\mathrm{cr}}$ on $\xi$ diminished for large values of $\xi$ and beyond a certain value of $\xi$, the value of $\lambda_{\text {cr }}$ becomes independent of the boundary conditions on the loaded edges and depends only on the boundary conditions on the unloaded edges. The prediction of this independence of $\lambda_{\text {cr }}$ at large $\xi$ added confidence to the numerical procedures adopted in the present work. Table 1 summarizes the limiting values of $\lambda_{\mathrm{cr}}$ as the plate's aspect ratio $\xi \rightarrow \infty$ for the case of an isotropic material.

An interesting feature of the buckling behavior of this problem is its symmetry. For a unilaterally constrained plate having homogeneous boundary conditions, the out-of-plane buckling deformation field $w(x, y)$ is a symmetric function with respect to the center line $x=\xi / 2$. This feature aids in the selection of the admissible displacement functions in (8) such that only the symmetric ones are retained and all others discarded due to their vanishing contribution to the governing equations.

Since the formulation covers material models other than isotropic, example problems for two types of specially orthotropic materials (designated A and B) were also studied. The properties of these materials are tabulated in Table 2. The results obtained via the present formulation for unilateral buckling of plates made of these materials are summarized in Fig. 7. For large aspect ratios the results for buckling loads are seen to converge to certain fixed values and when compared to previous results (Shahwan and Waas, 1991), are found to be in very good agreement.

In Fig. 8, we show typical plots for the evolution of the buckle displacements as a function of applied load for an isotropic plate of aspect ratio three. In the case, the plate was subjected to a uniformly distributed positive transverse pressure loading. Thus, at zero axial load, the plate deformation is the linear response to the pressure load.

\section{CONCLUSIONS}

The problem of buckling of unilaterally constrained, finite, rectangular plates was investigated. The plates were modeled along the lines of classical plate theory employing the Kirchhoff-Love hypothesis. The presence of a unilateral constraint was accounted for through the use of a nonlinear elastic foundation model that exhibits a deformation sign dependent force-displacement relation. Using Galerkin's method, the resulting system of governing nonlinear algebraic equations was solved iteratively. Different boundary conditions were considered and the results for some boundary conditions were compared and shown to be in good agreement with "exact" results available in the literature (Seide, 1958; Shahwan and Waas, 1991). The effect of the presence of a transvere load $Q$ was investigated and it was found that the plate's load-displacement $\left(\lambda-\left(\|\mathbf{A}\| /\left\|\mathbf{A}_{0}\right\|\right)\right)$ curve

Table 2. Normalized bending properties for the three types of materials used in this study

\begin{tabular}{cccccc}
\hline Material & $D_{12}$ & $D_{16}$ & $D_{22}$ & $D_{26}$ & $D_{66}$ \\
\hline Isotropic & 0.33 & 0.00 & 1.00 & 0.00 & 0.33 \\
Orthotropic (A) & 0.26 & 0.00 & 0.38 & 0.00 & 0.27 \\
Orthotropic (B) & 0.67 & 0.00 & 2.61 & 0.00 & 0.70 \\
\hline
\end{tabular}




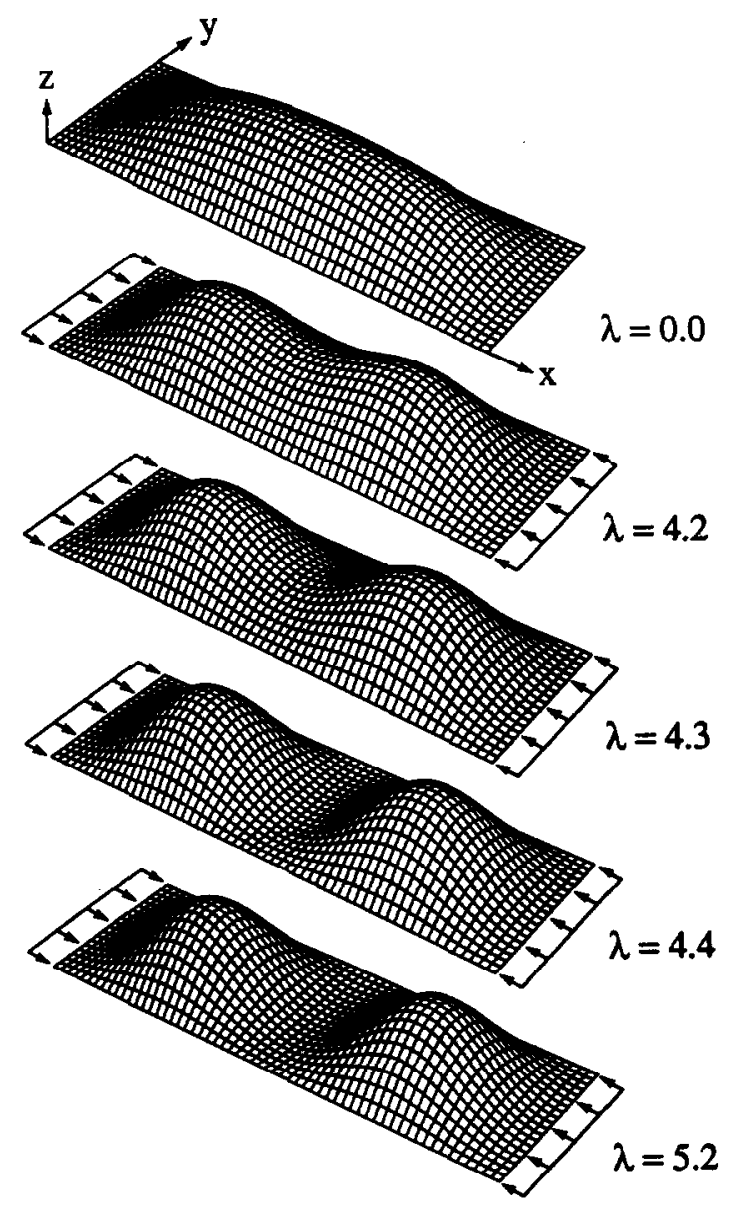

Fig. 8. Deformation of a "ccss" isotropic plate of aspect ratio $\xi=3$, under a uniform positive transverse loading and at different levels of inplane loading. The deformations are exaggerated for clarity.

can depend on the sign and distribution of $Q$, while the buckling load parameter $\left(\lambda_{\mathrm{cr}}\right)$ was found to be independent of $Q$. Different material orthotropy was also investigated and results for the buckling load were presented and were found to compare favorably with results reported previously. The present study has demonstrated the validity of using such foundation models in the buckling analysis of unilaterally constrained rectangular plates. Since the formulation is quite general, extensions to study the unilaterally constrained buckling problem of thin film delaminations of arbitrary planform shape in compressively loaded laminates is currently being pursued. Obtaining a solution to this latter problem was our initial motivation for developing the methodology presented here.

Acknowledgements - The authors are appreciative of the financial support received through AFOSR Initiation Grant RI-B-91-06 awarded through the Engineering Foundation in the early stages of this work. The financial support from the ONR Mechanics Division to (AMW) is gratefully acknowledged. Dr Yapa D. S. Rajapakse is the ONR program monitor.

\section{REFERENCES}

Allan, T. (1968). One-way buckling of a compressed strip under lateral loading. J. Mech. Engng Sci. 10(2), 173181.

Anderson, W. F. (1972). Buckling of a strip on a rigid half-space with a transverse pressure: Wrinkling of a nonbonded cladding. Proceedings of the First International Conference on "Structural Mechanics in Reactor Technology," Vol. 2, Part C, pp. 123-144, Berlin.

Barbero, E. J. and Reddy, J. N. (1991). Modeling of delamination in composite laminates using a layer-wise plate theory. Int. J. Solids Structures 28(3), 373-388. 
Benson, R. (1991). Plate tenting with a one-sided constraint. J. Appl. Mech. 58, $484-492$

Bottega, W. J. and Maewal, A. (1983). Delamination buckling and growth in laminates. J. Appl. Mech. 50, 184-189.

Brunelle, E. J. and Oyibo, G. A. (1983). Generic buckling curves for specially orthotropic rectangular plates. $A I A A J l 21(8), 1150-1156$.

Bruno, D. and Grimaldi, A. (1990). Delamination failure of layered composite plates loaded in compression. Int. J. Solids Structures 26(3), 313-330.

Burgess, I. W. (1971a). On the equilibrium and stability of discrete one-way structural systems. Int. J. Solids Structures 7, 667683 .

Burgess, I. W. (1971b). The buckling of a radially constrained imperfect circular ring. Int. J. Mech. Sci. 13, 741753.

Celep, Z. (1988). Rectangular plates resting on tensionless elastic foundations. J. Engng Mech. 114(12), 20832092.

Chai, H. (1990a). Buckling and post-buckling behavior of elliptical plates: Part I-Analysis. J. Appl. Mech. 57, $981-988$.

Chai, H. (1990b). Buckling and post-buckling behavior of elliptical plates : Part II-Results. J. Appl. Mech. 57, 989-994.

Chai, H. and Babcock, C. (1985). Two dimensional modeling of compressive failure in delaminated laminates. $J$. Compos. Mater. 19, 67-98.

Chai, H., Babcock, C. and Knauss, W. G. (1981). One dimensional modeling of failure in laminated plates by delamination buckling. Int. J. Solids Structures 17(11), 1069-1083.

Do, C. (1976). The buckling of a thin elastic plate subjected to unilateral conditions. In Applications of Methods of Functional Analysis to Problems of Mechanics, Lecture Notes in Mathematics (Edited by P. Germain and B. Naijroles), Vol. 503. Springer, Berlin.

Do, C. (1977). Bifurcation theory for elastic plates subjected to unilateral conditions. J. Math. Anal. Appl. 60, 435- 448.

Farshad, M. and Shahinpoor, M. (1972). Beams on bilinear elastic foundations. Int. J. Mech. Sci. 14, 441-445.

$\mathrm{Fu}, \mathrm{L}$. and Waas, A. M. (1992). Buckling of polar and rectilinearly orthotropic annuli under uniform internal or external pressure loading. Compos. Struct. 22, 47-57.

Gerard, G. (1962). Introduction to Structural Stability Theory. McGraw-Hill.

Gerard, G. and Becker, H. (1957). Handbook of Structural Stability. I : Buckling of Flat Plates. NACA TN 3781.

Hobbs, R. E. (1985). Discussion of: "Buckling and postbuckling of the lying sheet by C. Y. Wang". Int. J. Solids Structures 21(4), 423-424. Author's reply to R. E. Hobbs (1985), 21(4), 425.

Hobbs, R. E. (1990). Axisymmeric ujpheaval buckling of a heavy sheet. J. Appl. Mech. 57, 472-474.

Kubrusly, R. S. and Oden, J. T. (1981). Nonlinear eigenvalue problems characterized by variational inequalities with applications to the postbuckling of unilaterally-supported plates. Nonlin. Anal. Theory, Methods and Applications 5(12), 1265-1284.

Majumdar, S. (1971). Buckling of a thin annular plate under uniform compression. AIAA Jl, 9(9), 1701-1707.

Naumann, J. (1975). On some unilateral boundary value problems for the von Kármán equations, Part I: The Coercive case. Aplikace Matematiky 20, 96-125.

Nauman, J. (1977). On some unilateral boundary value problems in nonlinear plate theory. Beiträge zur Analysis $10,119134$.

Ohtake, K., Oden, J. T. and Kikuchi, N. (1980a). Analysis of certain unilateral problems in von Kármán plate theory by a penalty method. Part $1:$ A variational principle with penalty. Comput. Meth. Appl. Mech. Engng 24, 187-213.

Ohtake, K., Oden, J. T. and Kikuchi, N. (1980b). Analysis of certain unilateral problems in von Kármán plate theory by a penalty method. Part 2: Approximation and numerical analysis. Comput. Meth. Appl. Mech. Engng 24, 317-337.

Plaut, R. H. and Mróz, Z. (1992). Uni-directional buckling of a pinned elastica with external pressure. Int. J. Solids Structures 29(16), 2091-2100.

Powell, M. J. D. (1970). A hybrid method for nonlinear equations. In Numerical Methods for Nonlinear Algebraic Equations (Edited by P. Rabinowitz), Chapter 6, pp. 87-114. Gordon and Breach, New York.

Roorda, J. (1988). Buckles, bulges and blow-ups. Appl. Solid Mech.-2 (Edited by A. S. Tooth and J. Spence), pp. 347-380. Elsevier, London.

Sallam, S. and Simitses, G. J. (1985). Delamination buckling and growth of flat, cross-ply laminates. Compos. Struct. 4, 361-381.

Seide, P. (1958). Compressive buckling of a long simply supported plate on an elastic foundation. J. Aeronaut. Sci., 382-392.

Shahwan, K. and Waas, A. (1991). Elastic buckling of infinitely long specially orthotropic plates on tensionless foundations. J. Engng Mater. Technol. 113, 396-403. (Errata, 114, 124-125.)

Soong, T. C. and Choi, I. (1986). An elastica that involves continuous and multiple discrete contacts with a boundary. Int. J. Mech. Sci. 28(1), 1-10.

Stein, E. and Wriggers, P. (1984). Stability of rods with unilateral constraints, a finite element solution. Comput. Struct. 19(1-2), $205-211$.

Tsai, N. and Westmann, R. (1967). Beams on tensionless foundation. J. Engng Mech. Div. 93, 1-12.

Villaggio, P. (1979). Buckling under unilateral constraints. Int. J. Solids Structures 15, 193-201.

Wang, C. Y. (1984a). On symmetric buckling of a finite flat lying heavy sheet. J. Appl. Mech. 51, 278-282.

Wang, C. Y. (1984b). Buckling and postbuckling of the lying sheet. Int. J. Solids Structures 20(4), 351-358.

Yun, H. D. and Kyriakides, S. (1983). Localized buckling of a heavy beam on a contacting surface : A model for beam mode buckling of buried pipelines. Engineering Mechanics Research Laboratory, EMRL report No. 83/5, Dept. of Aerospace Engineering and Engineering Mechanics, The University of Texas, Austin, TX. 